\title{
Will the leaking stop?
}

\author{
Edward Y. Sako, MD, PhD
}

\author{
From the Department of Cardiothoracic Surgery, University of Texas Health Science Center at San Antonio, Tex. \\ Disclosures: E.Y.S. has a financial relationship with Medtronic, Inc. \\ Received for publication Dec 17, 2015; accepted for publication Dec 17, 2015; available ahead of print Jan 12, \\ 2016. \\ Address for reprints: Edward Y. Sako, MD, PhD, Department of Cardiothoracic Surgery, University of Texas \\ Health Science Center at San Antonio, Mail Code 7841, 7703 Floyd Curl Dr, San Antonio, TX 78229-3900 \\ (E-mail: sako@uthscsa.edu). \\ J Thorac Cardiovasc Surg 2016;151:1043 \\ $0022-5223 / \$ 36.00$ \\ Copyright (c) 2016 by The American Association for Thoracic Surgery \\ http://dx.doi.org/10.1016/j.jtcvs.2015.12.037
}

In any given cardiac operation, the need to resolve all the pathology needs to be balanced against the price that is paid by making the procedure more complex with the attendant longer pump and crossclamp times. A particularly vexing situation arises when it is possible that some of the pathology may improve once the major problems have been addressed. The fate of functional mitral regurgitation (MR) after coronary artery revascularization has long been debated. ${ }^{1}$

A similar situation arises when MR accompanies aortic stenosis. In cases in which there is no obvious mitral structural abnormalities such as leaflet prolapse, will the reduction in left ventricular intracavitary pressures and remodeling after aortic valve replacement lead to improvement in MR? In this issue of the Journal, Schubert and colleagues $^{2}$ attempt to answer this question. They undertook a retrospective analysis of more than 400 patients who underwent aortic valve replacement with coexistent mild or moderate MR. They found that in the majority of patients, there was some initial improvement in MR after aortic valve replacement, but that over time this improvement regressed and in some cases the MR worsened. Most important, the presence of residual MR could not be shown to have an effect on medium-term survival. Also, it is important to note that this group surgically treated all instances of associated severe MR and none of the cases of mild or moderate during this study period.

As with most studies of this type, there are factors that limit the immediate applicability to one's own practice. Readily acknowledged are the problems of a retrospective, single institution study and the hazards inherent in assessing and quantifying MR. Most significant is the timing of the postoperative echocardiograms. A total of 903 postoperative echocardiograms were reviewed for the study. A total of $636(70 \%)$ of them were performed in the first 6 months postoperatively. The distribution of the remainder makes it clear that a large number of patients probably did not undergo echocardiography beyond that period, reducing the

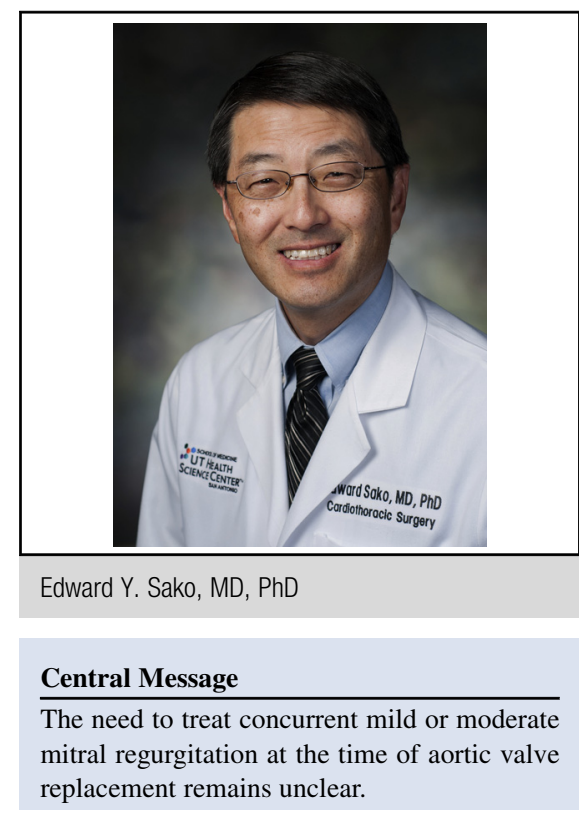

See Article page 1032.

power of any medium-term findings. Furthermore, as admitted in the article, there is no protocol for postoperative echocardiograms in their institution. Many of the echocardiograms used were the intraoperative postprocedure transesophageal echocardiograms. Given the changing hemodynamics, these studies may not represent any clinically significant findings. Echocardiograms obtained later may have been biased because they may have been weighted toward patients who were having clinical problems. This further weakens any findings related to the postoperative echocardiograms and the relationship to survival.

So although the questions raised are important, it is still unclear as to what the definitive answer is in this situation. The finding that there was no statistical difference in 5-year mortality between those with mild MR and those with moderate MR may be heartening to some but does not address all the considerations. As is often the case, more study is needed.

\section{References}

1. Aklog L, Filsoufi F, Flores KQ, Chen RH, Cohn LH, Nathan NS, et al. Does coronary artery bypass grafting alone correct moderate ischemic mitral regurgitation? Circulation. 2001;104:I68-75.

2. Schubert SA, Yarboro LT, Madala S, Ayunipudi K, Kron IL, Kern JA, et al. Natural history of coexistent mitral regurgitation following aortic valve replacement. $J$ Thorac Cardiovasc Surg. 2016;151:1032-42.e1. 